\title{
Prevalencia endoparasitaria de serpientes Bothrops asper (Garman, 1884) y Bothrops atrox (Linnaeus, 1758) en condiciones de cautividad en el Ecuador
}

\author{
María del Carmen Terán $Z^{1}$, Mariana Estrada $V^{2}$ y Manolo Puente ${ }^{2}$ \\ ${ }^{1}$ Empresa Pública de Fármacos ENFARMA \\ ${ }^{2}$ Instituto Nacional de Salud Pública e Investigación INSPI Guayaquil, Ecuador \\ macateza11@hotmail.com_Recibido: 2014-02-27; aceptado: 2014-06-14
}

\section{RESUMEN.-}

Un total de 75 serpientes venenosas pertenecientes al proceso de producción de sueros antiofídicos nacionales de la ciudad de Guayaquil, Ecuador fueron analizadas; 48 B. asper y 27 B. atrox muertas en condiciones de cautiverio desde el 2010 al 2013. Los informes anatomopatológicos señalaron el estado caquéctico de la mayor parte de los ofidios ingresados, así como también la observación de lesiones encontradas a nivel de órganos. Los análisis parasitológicos evidenciaron en ambas especies de ofidios la presencia de endoparásitos y la prevalencia obtenida sugiere la aplicación de medidas de control prioritarias para coccidias y nemátodos en serpientes cautivas.

PALABRAS CLAVES: anatomopatológico, Bothrops, endoparásitos, parasitológico, serpientes

\section{ABSTRACT.-}

A total of 75 venomous snakes belonging to the national production process of antivenom in Guayaquil - Ecuador were analyzed; 48 B. asper and 27 B. atrox dead in captivity from 2010 to 2013. The anatomopatologic reports said the cachetic state admitted most snakes, as well as the observation of lesions found in organs. Parasitological examinations showed both species of snakes in the presence and prevalence of endoparasites obtained suggests the application of control measures priority coccidia and nematodes in captive snakes.

KEYWORDS: Bothrops, endoparasites, parasitology, pathological, snakes

\section{INTRODUCCIÓN}

El género Bothrops comprende más de 37 especies comúnmente conocidas como "cabezas de lanza" (Campbell y Lamar, 2004), algunas de ellas con aspectos de su historia natural ya documentados (Mahmood et al., 2009; Oliveira y Martins, 2001).

Bothrops asper (Garman, 1884) es una especie de tamaño grande con hábitos terrestres (Meza-
Ramos et al., 2010), sus mordeduras causan hemorragias sistémicas, descenso de la temperatura (señal de shock), infección y necrosis (Prado-Franceschi y Hyslop, 2002). Además, B. atrox (Linnaeus, 1758) del oriente de Ecuador, es la mayor causa de accidentes ofídicos y se ha registrado que es la causante de prácticamente todas las mordeduras en las tribus Waorani, donde la mayoría de adultos han sido mordidos por lo menos una vez (Smalligan et al, 2004). Al 
ser ambas especies consideradas de gran importancia médica, resulta indispensable su manejo en cautividad para la producción de sueros antiofídicos nacionales (WHO, 2010).

Es conocido en los reptiles que el estrés de la cautividad puede conducir a enfermedades graves (Mihalca, 2010; Santos et al., 2008; QuesadaMorúa, 2005; Modrý et al., 2001), y en estudios provenientes de serpientes en cautiverio, el endoparasitismo ha sido una de las enfermedades mayormente reportadas (Pinto et al., 2010; Junker et al., 2009; Seghetti y Traversa, 2007) encontrándose entre estos hallazgos: nemátodos (Silva et al., 2007; Siqueira et al., 2005), céstodos (Gómez-Puerta, 2011; Sánchez et al., 2004), pentastómidos (Ayinmode et al., 2010; Gárate et al., 2007), protozoos (Matsubara et al., 2002), entre otros.

Para adquirir información sobre la fauna parasitológica, el diagnóstico de la infección parasitaria se consigue comúnmente al revelar elementos parásitos microscópicos mediante técnicas de rutina (Radhakrishnan et al., 2009), o mejoradas para el caso de los reptiles (Rinaldi et al., 2012).

También es muy útil la realización de necropsias que permitan la observación macroscópica de individuos adultos presentes así como también, lesiones anatomopatológicas relacionadas (Tappe et al., 2011).

El objetivo del presente estudio es investigar la prevalencia de fauna endoparasitaria existente en serpientes ecuatorianas Bothrops asper y Bothrops atrox sometidas a condiciones de cautiverio.

\section{MATERIALES Y MÉTODOS}

Se trasladaron 75 serpientes pertenecientes al Subproceso Serpentario de la Empresa Pública de Fármacos ENFARMA EP, al área de Patología Veterinaria del Instituto Nacional de Salud Pública e Investigación INSPI para la respectiva necropsia, 48 B. asper y 27 B. atrox muertas en condiciones de cautiverio del año 2010 al 2013; las mismas formaban parte del stock de serpientes utilizadas para el proceso de producción de sueros antiofídicos a cargo de la misma institución, en la ciudad de Guayaquil.

Las serpientes fueron colectadas en distintas provincias del país. Posteriormente fueron acondicionadas al cautiverio recibieron agua y alimento basados en el suministro de ratones (Mus musculus) ofrecidos de acuerdo con el tamaño de los especímenes y no recibieron en ningún momento aplicación de tratamientos parasitarios.

Durante las necropsias realizadas, se obtuvieron muestras de intestinos para los respectivos análisis parasitarios, estos se realizaron mediante frotis directo y técnica de flotación por solución salina sobresaturada. La lectura se realizó con ayuda de microscopia óptica y el diagnóstico inmediato de los parásitos se obtuvo con la aplicación de patrones de identificación. Los resultados anatomopatológicos y parasitológicos fueron emitidos a través de informes oficiales.

\section{RESULTADOS}

Los informes anatomopatológicos manifestaron el estado caquéctico en el cual se encontraban la mayor parte de las serpientes ingresadas. En las necropsias se observaron lesiones macroscópicas en los órganos; corazón flácido y congestionado, pulmón hemorrágico o congestionado, hígado friable con parénquima de coloración negruzca y en muy pocos casos con áreas hemorrágicas, los intestinos presentaron mucosa engrosada, congestionada o hemorrágica; además de heces de consistencia pastosa o líquida de color blanquecino en unos casos y en otros amarillento. Se encontraron vermes adultos de Armillifer sp., en los pulmones de varios ejemplares de $B$. asper y B. atrox.

Los análisis parasitarios identificaron la presencia de uno, dos o hasta tres endoparásitos diferentes, en estado adulto o huevo, en un mismo ofidio. La prevalencia fue determinada para ambas especies: en $B$. asper las Coccidias se presentaron en un $60 \%$, Strongyloides sp. $38 \%$, Oxyuris sp. $23 \%$, Armillifer sp. 15\%, Ascaris sp. 6\%, Hymenolepis sp., Balantidium sp. 4\%, y Trichuris sp. 2\% (Fig. 1). 
En B. atrox no se manifestaron Ascaris sp., Balantidium sp. y Trichuris sp., sin embargo la prevalencia en las otras especies de endoparásitos, fue la siguiente: Coccidias $81 \%$, Strongyloides sp. 33\%, Armillifer sp. 22\%, Oxyuris sp. 19\% e Hymenolepis sp. 7\% (Fig. 2).

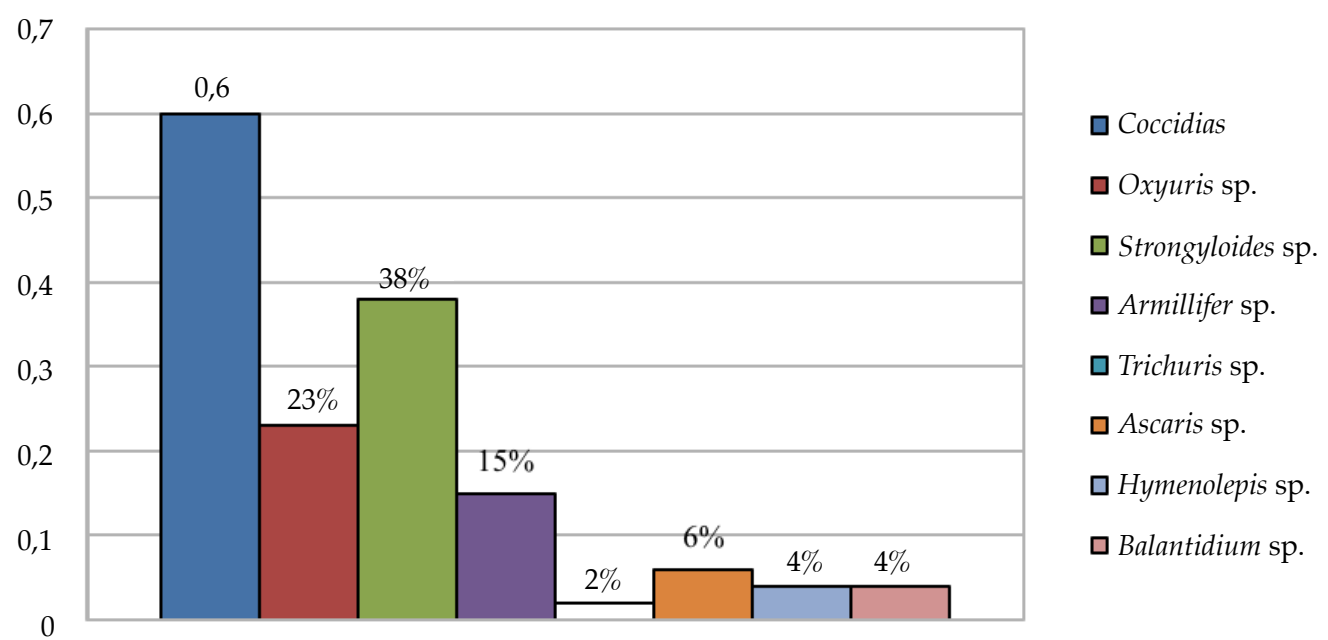

Fig 1. Prevalencia endoparasitaria de serpientes Bothrops asper en cautiverio.

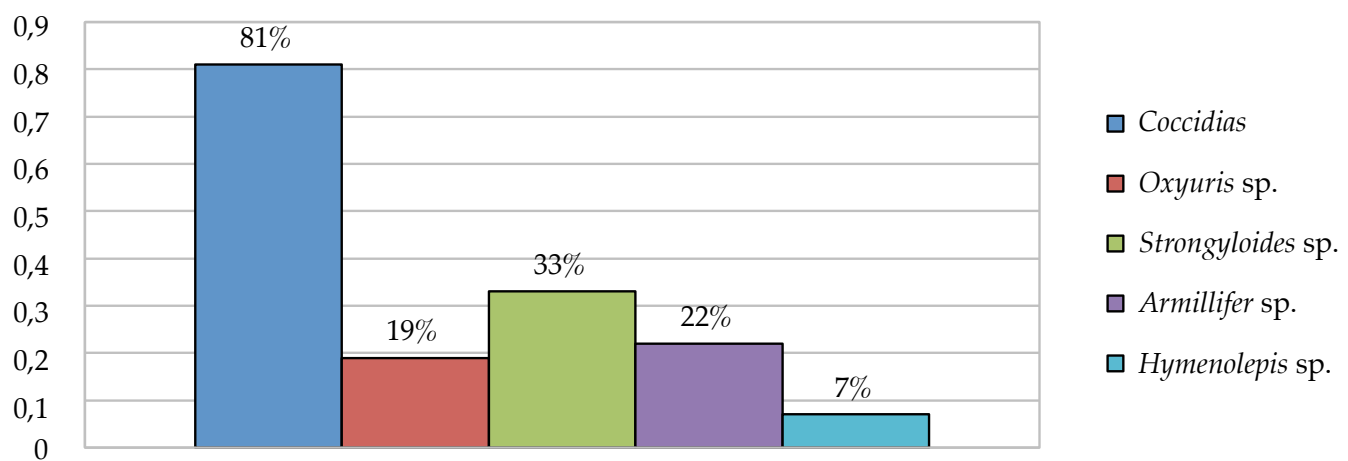

Fig 2. Prevalencia endoparasitaria de serpientes Bothrops atrox en cautiverio.

\section{DISCUSIÓN}

El estado caquéctico encontrado en los especímenes del presente estudio es característico de serpientes altamente parasitadas. Junker et al. (2009) reportaron 73 nemátodos gastrointestinales del esófago y estómago de una serpiente Pseudaspis cana, y consideraron que la cantidad de nemátodos encontrados pudo haber contribuido con el estado caquéctico, caracterizado por pérdida de peso y deshidratación en el cual se encontraba el ejemplar.

Serpientes y lagartos en cautividad pueden albergar una gran variedad de parásitos y son los más comúnmente encontrados protozoos y nemátodos (Rinaldi et al., 2012). En el presente estudio, B. atrox tuvo una prevalencia del $81 \%$ de protozoarios, mucho más alta comparada con el $64 \%$ de prevalencia en B. asper (Coccidias $60 \%$ y Balantidium sp. 4\%). Ambos endoparásitos están ampliamente distribuidos en los reptiles en cautiverio (Matsubara et al., 2002; Sátorhelyi y Sréter, 1993). El ciclo de vida de las coccidias es en su mayoría directo y son altamente patógenos, pudiendo causar daños graves (Schneller y Pantchev, 2008), por lo cual el estrés de la cautividad pudo haber contribuido con estos hallazgos (Matsubara et al., 2002), que a su vez se encuentran en común acuerdo con los ya reportados por 
otros autores (Xiao et al., 2004; Mihalca, 2002). Los informes anatomopatológicos de las serpientes infestadas por coccidias supieron manifestar la presencia de heces de consistencia pastosa o líquida de color amarillento o blanquecino, posiblemente como signo clínico común en la aparición de este tipo de protozoos.

En nemátodos el parasitismo abrumador es frecuente cuando la falta de higiene da lugar a ambientes altamente contaminados (Merck, 2007). En nuestra investigación, Strongyloides sp., con un 38\% de prevalencia en B. asper y $33 \%$ en B. atrox, fueron los nemátodos más frecuentes seguidos de Oxyuris sp., con un $23 \%$ y $19 \%$, respectivamente. Así mismo, Rataj et al. (2011) ponen de manifiesto que en la investigación realizada a 55 serpientes criadas en condiciones de cautiverio, los strongilidos se encontraron de igual manera con una prevalencia mayoritaria en animales mantenidos tanto en condiciones silvestres (Breves et al., 2011) como en cautividad (Loukopoulus et al., 2007; Araújo et al, 1999).

La exploración fecal reveló la presencia de huevos de Ascaris sp., y Trichuris sp,. en B. asper, ambos nemátodos han sido identificados en serpientes a pesar de afectar mayormente a roedores (Rinaldi et al., 2012 y Rataj et al., 2011), situación que podría relacionarse con el sistema de alimentación basada en ratones que se les proporcionó durante el cautiverio; sin embargo, se conoce también que en condiciones naturales la predación de roedores en esta especie también es muy común (Boada et al., 2005). Varias serpientes regurgitaron el alimento parcialmente digerido y en otro casos el parásito adulto, siendo este un signo clínico característico de la infestación por áscaris (Merck, 2007).

Entre los pentastómidos, Armillifer sp. ha sido encontrado adherido a los pulmones de serpientes destruyendo y deteriorando la función pulmonar por perforación del tejido produciendo hemorragias (Ayinmode et al., 2010). La patología asociada a la presencia de estos vermes en ambas especies de serpientes incluyó pulmones congestionados o hemorrágicos, la prevalencia de estos pentastómidos mayormente encontrada en B. atrox posiblemente es una de las causas que dificulta su permanencia en cautiverio.

La prevalencia de huevos de Hymenolepis sp., en serpientes de ambas especies resultó ser relativamente baja, situación que se considera asociada al complejo ciclo de vida de los céstodos y la extensión geográfica restringida de los hospedadores intermediarios que limita el número de casos en los reptiles cautivos (Merck, 2007). Se ha evidenciado el hallazgo de céstodos en serpientes (Gómez-Puerta, 2011) y particularmente en crías de Boa constrictor se ha observado que la teniasis es temprana la misma que puede ser la causa de morbilidad y mortalidad en animales jóvenes y adultos cautivos (Sánchez et al., 2004).

Los resultados obtenidos en el presente estudio informan sobre la existencia de fauna endoparasitaria en B. asper y B. atrox muertas durante la cautividad, así como lo altos índices de infección por coccidias y nemátodos que afectan a estos ofidios, con lo cual sugiere la necesidad de aplicación de intensas medidas que ayuden al control de estos endoparásitos.

\section{REFERENCIAS BIBLIOGRAFICAS}

Araújo T, Moro L, Lúcia M, Golloubeff B, Vasconcelo AC. 1999. Ocorrência de alguns endo e ectoparasitos no serpentário da UNIFENAS - Universidade de AlfenasMG. Brazilian Journal of Veterinary Research and Animal Science, 36 (1):1922.

Ayinmode AB, Adedokun AO, Aina A y Taiwo V. 2010. The zoonotic implications of pentastomiasis in the royal python (python regius). Ghana Medical Journal, 44(3).

Boada C, Freire Lascano A, Salazar-V D y Kuch U. 2005. The diet of Bothrops asper (Garman, 1888) in the pacific lowlands or Ecuador. Herpetozoa, 18: 77-83

Breves P, Porto M, Pissinatti A, Luz D, Menezes RC. 2011. Helmintos oxiuridae parasitos de Iguana iguana (Squamata, Lacertilia, Iguanidae) procedentes do Brasil. Arquivo 
Brasileiro de Medicina Veterinária y Zootecnia, 63 (6): 1574-1578.

Campbell JA, Lamar WW. 2004. The Venomous Reptiles of the Western Hemisphere. Comstock Publishing Associates, Cornell University Press. Ithaca NY, 774 pp.

Gárate C, Naupay I, Suyo L, Colquichagua A, Rodríguez Q y Yarlequé Ch. 2007. Identificación de Porocephalus stilessi (Pentastomida) en la serpiente peruana Lachesis muta. Revista de Investigaciones Veterinarias del Perú. 18 (2): 89-93.

Gómez-Puerta L. 2011. Primer registro de Crepidobothrium gerrardii (Cestoda: Proteocephalidae) en el Perú. Revista Peruana de Biología, 18(3): 387-388.

Junker K, Lane EP, Dlamini B, Kotze A y Boomker J. 2009. Post mortem identification of Kalicephalus colubri colubri (Nematoda: Diaphanocephalidae) in a captive mole snake (Pseudapis cana) in South Africa. Journal of the South African Veterinary Association, 80: 54-56.

Loukopoulos P, Komnenou A, Papadopoulos E. et al. 2007. Lethal Ozolaimus megatyphlon infection in a green iguana (Iguana iguana rhinolopa). The Journal of Zoo and Wildlife Medicine, 38: 131-134.

Mahmood S, Wasko D y Lamar W. 2009. Natural history of the terciopelo Bothrops asper (Serpentes: Viperidae) in Costa Rica. Toxicon, 54: 904-922.

Matsubara A, Da Silva R, Mascarini L, Barrella T y Magalhães CA. 2002. Occurrence of Cryptosporidium (Apicomplexa, Cryptosporidiidae) in Crotalus durissus terrificus (Serpentes, Viperidae) in Brazil. Memorias del Instituto Oswaldo Cruz, 97(6): 779-781.

Merck C. 2007. Manual Merck de Veterinaria. Sexta Edición. Merck \& CO INC Whitehouse Station, NJ USA. 2682 pp.
Meza-Ramos P, Almendáriz A y Yánez-Muñoz MH. 2010. Datos sobre la dieta de Bothriechis schlegelii (Berthold, 1846) (Serpentes-Viperidae) en el Occidente del Ecuador. Boletín Técnico 9, Serie Zoológica 6: 15-18.

Mihalca AD, Miclaus V y Lefkaditis M. 2010. Pulmonary lesions caused by the nematode Rhabdias fuscovenosa in a grass snake, Natrix natrix. Journal of Wildlife Diseases, 46: 678-681.

Mihalca AD. 2002. Un studiu coproparazitologic la reptile captive. Scientia Parasitologica 3: 159-163.

Modrý D, Daszak P, Volf J, Veselý M, Ball SJ y Koudela B. 2001. Five new species ofcoccidia (Apicomplexa: Eimeriidae) from Madagascan chamaleons (Sauria: Chamaeleonidae). Systematic Parasitology, 48: 117-123.

Oliveira ME y Martins M. 2001. When and where to find a pitviper: activity patterns and habitat use of the lancehead, Bothrops atrox, in central Amazonia, Brazil. Herpetological Natural History, 8 (2):101-110.

Pinto RM, Muniz-Pereira LC, Panizzutti, MMH \& Noronha D. 2010. Nematode parasites of the neotropical rattlesnake, Crotalus durissus linnaeus, 1758 (Ophidia, viperidae) from Brazil: new records and general aspects. Neotropical Helminthology, 4 (2): 137-147.

Prado-Franceschi J y Hyslop S. 2002. Soutn American colubrid envenomations. Journal of Toxicology, Toxin Reviews 21(12):117-158.

Quesada-Morúa R. 2005. Hallazgos Anatomohistopatológicos en Serpientes en Cautiverio en Costa Rica. Tesis. Escuela de Medicina Veterinaria, Universidad Nacional, Heredia, Costa Rica, 81 pp. 
Radhakrishnan S, Kurup SP y Banerjee PS. 2009. Endoparasitism in captive wild-caught snakes indigenous to Kerala, India. Zoo Biology, 28: 253-258.

Rataj A, Lindtner-Knific R, Vlahović K, Mavri U y Dovč A. 2011. Parasites in pet reptiles. Acta Veterinaria Scandinavica, 53:33.

Rinaldi L, Mihalca AD, Cirillo R, Maurelli MP, Montesano M, Capasso M y Cringoli G. 2012. FLOTAC can detect parasitic and pseudoparasitic elements in reptiles. Experimental Parasitology, 130: 282-284.

Sánchez P, Tantaleán V, Richards G y Gálvez C. 2004. Parásitos helmintos en Boa constrictor, Epicrates cenchria y Corallus caninus (Ophidia: Boidae) criadas en cautiverio. Revista de Investigaciones Veterinarias del Perú, 15 (2): 166-169.

Santos KR, Takahira RK, Rall VLM, Calderón C, Sequeira JL y Silva RJ. 2008. Pulmonary, microbiological and hematological changes in Crotalus durissus terrificus (Serpentes, Viperidae) parasitized by nematodes of the genus Rhabdias (Nematoda, Rhabdiasidae). Arquivo Brasileiro de Medicina Veterinária e Zootecnia, 60 (3): 667-674.

Sátorhelyi T y Sréter T.1993. Studies on internal parasites of tortoises. Parasitología Hungarica, 26: 51-55.

Schneller P y Pantchev N. 2008. Parasitology in Snakes, Lizards and Chelonians. A Husbandry Guide, Edition Chimaira, Frankfurt am Main, Germany. 205 pp.

Seghetti M y Traversa D. 2007. Indagine parassitologica in boidae allevati in cattivita in Italia centrale. Large Animal Reviews, 13: 107-112.
Silva RJ, Nogueira MF, Barrella TH y Takaira RK. 2007. Frequency of rhabdiasid nematodes in wild Crotalus durissus terrificus (serpentes, viperidae) from Botucatu Region, São Paulo State, Brazil. Journal of Venomous Animals and Toxins including Tropical Diseases, 13 (1): 122-129.

Siqueira R, Panizzutti HM, Muniz-Pereira C y Pinto M. 2005. Description of a new ascaridoid parasite of Bothrops jararaca Wied (Reptilia, Ophidia) in Brazil. Revista Brasileira de Zoología, 22(3): 587-590.

Smalligan R, Brito N, Laing GD, Mertz BL, Manock S, Maudlin J, Quist B, Holland G, Nelson S, Lalloo DG, Rivadeneira G, Barragan ME, DollEy, Eddleston M, Warrel DA, y Theakston RDG. 2004. Crotaline snake bite in the Ecuadorian Amazon: Randomiseddouble blind comparative trial of three South American polyspecific antivenoms. British Medical Journal, 329: 1129.

Tappe D, Meyer M, Oesterlein A, Jaye A, Frosch M, Schoen C y Pantchev N. 2011. Transmission of Armillifer armillatus Ova at Snake Farm,The Gambia, West Africa. Emerging Infectious Diseases, 17(2).

World Health Organization (WHO). 2010. Guidelines for the production control and regulation of Snake antivenom Inmunoglobulins.Geneva, Switzerland. 134 pp.

Xiao L, Ryan M, GraczykK, Limor J, Li L, Kombert M, Junge R, Sulaiman IM, Arrowood MJ, Koudela B, Modrý D y Lal AA. 2004. Genetic diversity of Cryptosporidium sp. in captive reptiles. Applied and Environmental Microbiology, 70: 891-898 